\title{
ARTÍCULOS
}

\section{LA RETÓRICA DEL RETORNO: MINGMA O LAS CONTRADICCIONES DEL DESARROLLO EN NEPAL*}

\author{
THE RHETORIC OF RETURN: MINGMA OR THE CONTRADICTIONS OF \\ DEVELOPMENT IN NEPAL
}

\author{
Alba Castellsagué ${ }^{1}$ \\ Grupo EMIGRA (CER-Migraciones), Universidad Autónoma de Barcelona.
}

Recibido: 14 de febrero de 2019; Aprobado: 20 de marzo de 2019

Cómo citar este artículo / Citation: Castellsagué, Alba. 2020. "La retórica del retorno: Mingma o las contradicciones del desarrollo en Nepal». Disparidades. Revista de Antropología 75(2): e025. doi: <https://doi.org/10.3989/dra.2020.025>.

RESUMEN: Marcharse a la ciudad o al extranjero a estudiar para después volver y contribuir al desarrollo del pueblo. Esta idea constituye lo que proponemos como retórica del retorno, un concepto polisémico y central en los discursos del desarrollo y la educación en Nepal. La trayectoria migratoria de Mingma, una joven sherpa que ha crecido en Sikkim (India), problematiza estas ideas a partir de una experiencia de retorno a Gaun (Nepal), el pueblo de su familia. A través de su narrativa nos adentramos en las negociaciones que le suponen sus ideales del desarrollo, su rol como profesora y su relación con la gente del pueblo. Los hallazgos más importantes muestran la estrecha interrelación entre los regímenes de movilidad y conocimiento en Nepal y evidencian la relevancia del género en el nexo movilidad-desarrollo y sus contradicciones.

PALABRAS CLAVE: Movilidad de retorno; Desarrollo; Género; Educación; Nepal.

ABSTRACT: Studying in the city or abroad and returning later to contribute to the village's development is the idea underlying what we consider as the rhetoric of return, a polysemic concept that is central to both development and educational discourses in Nepal. The case of Mingma, a Sherpa girl raised in Sikkim, and her experience of returning to her family village of Gaun (Nepal), problematizes this notion. Her narrative enables us to understand the negotiations arising from her ideals about development, her role as a teacher, and her relationships with the villagers. Findings show the link between mobility and knowledge regimes in Nepal, and demonstrate the relevance of a gender perspective in the mobility-development nexus and its contradictions.

KEYWORDS: Return mobility; Development; Gender; Education; Nepal.

Copyright: @ 2020 CSIC. Este es un artículo de acceso abierto distribuido bajo los términos de la licencia de uso y distribución Creative Commons Reconocimiento 4.0 Internacional (CC BY 4.0).

\footnotetext{
Parte de la investigación ha sido financiada por la Fundación Autónoma Solidaria (FAS-UAB): Convocatoria E2014-2015 / Beca E1415-01. Agradecimientos a Ms. Injina Panthi, ayudante de investigación en Nepal, y a la Dra. Sílvia Carrasco, por la supervisión y contribuciones al artículo.

1 Correo electrónico: alba.castellsague@uab.cat. ORCID iD: <https://orcid.org/0000-0002-7625-6876>.
} 


\section{INTRODUCCIÓN}

La educación en Nepal ocupa una posición central a nivel simbólico y en las políticas públicas. Los discursos hegemónicos del desarrollo han potenciado la educación como herramienta para salir de las condiciones de pobreza, especialmente en el caso de las mujeres y las niñas, que se (pre)suponen limitadas por las costumbres culturales tradicionales (Mohanty 1984; Tamang 2000). Tal conditio sine qua non para avanzar hacia la modernidad implica normalmente movilidad hacia Katmandú o el extranjero, ya que las oportunidades de escolarización en las zonas rurales son escasas y/o percibidas como de baja calidad. A dichos movimientos se les asocia una idea inespecífica de retorno, supuesto a contribuir con las habilidades adquiridas al desarrollo de los lugares y personas con menos oportunidades.

Pero el retorno es un fenómeno polisémico de compleja conceptualización (Pascual de Sans 1993; Sinatti 2015) que permite por lo menos tres aproximaciones. En primer lugar, el retorno como movimiento físico, dominante en los estudios sobre migraciones. De las aproximaciones neoclásicas que concebían lugares de origen y destino al transnacionalismo, que lo ha conceptualizado como parte de dinámicas de circularidad (Cavalcanti y Parella 2013) y un movimiento más en proyectos migratorios más amplios (Pascual de Sans 1983; Gil Araujo et al. 2013). El retorno ha ocupado un lugar central en la comprensión de las migraciones a escala global, identificando fenómenos como el mito del retorno, tan bien establecido como controvertido en las investigaciones de las últimas décadas (Madawi 1994; King 2000; Ali y Holden 2006; Carling et al. 2015). Pero el retorno no es solamente una vuelta -más o menos definitiva- a un lugar -considerado o no como origen-, sino también sus significados e implicaciones. En segundo lugar, el retorno desde una perspectiva de la economía política se refiere al tipo de devoluciones que las personas pueden hacer a partir de su experiencia de movilidad con la intención de mejorar la situación y/o el estatus personal o familiar, dado que "la narrativa de la migración como movilidad física está fuertemente vinculada a la promesa de movilidad social» (Oso, Sáiz-López y Cortés 2017: 296). La articulación de ambas movilidades cruzadas (Oso, Sáiz-López y Cortés 2017), se concreta en el nexo migración-desarrollo (Cortés, Fernández y Sanmartín 2007; Cortés 2009; Faist y Fauser 2011), en particular cuando se sitúan en un contexto global de relaciones Norte-Sur, fuer- temente politizadas en tanto que producto de circunstancias históricas, ideológicas y mediáticas (Appadurai 1996; Cortés 2009). Desde los años '90, los discursos hegemónicos y las políticas públicas promueven una visión positiva del nexo muy vinculada a las teorías clásicas de la modernización, entendiendo la movilidad como una contribución potencial al desarrollo de la familia, comunidad o país de origen (Cortés, Fernández y Sanmartín 2007; Cortés 2009; Faist y Fauser 2011; Cortés 2013). Tal explicación se ha convertido en premisa ideológica constitutiva del co-desarrollo (Cortés 2009; Gil Araujo et al. 2013) y ha destacado el migrante y sus capacidades adquiridas en y a través de la movilidad como agente de desarrollo, visión especialmente acentuada en el caso de las mujeres y las minorías (Bereményi y Carrasco 2017). Sin embargo, algunas investigaciones han problematizado dichas aproximaciones, señalando que se ha sobreestimado el impacto de la migración de retorno (King 2000; Sinatti 2015) y su carácter economicista y desarrollista (Cortés 2013; Vega 2016). En menor medida se han realizado críticas desde la perspectiva de género que, a pesar de ser un elemento bien establecido en la investigación sobre migraciones (Mora 2008; Oso y Ribas-Mateos 2012; Cortés 2015), no ha sido tan central en los análisis sobre el nexo migración-desarrollo (Piper 2008; Rao 2010; Cortés 2015).

Nuestra investigación no se centra tanto en el volver, sino en los significados y experiencias del retorno como proceso de (de)volver y contribuir a la comunidad, aplicando una perspectiva de género en el contexto nepalí. Nepal es un caso paradigmático de desarrollo o bikas, término específico para referirse a él. Construido semántica y simbólicamente en oposición a lo tradicional y lo rural (Pigg 1992 y 1993) el bikas está vinculado a la modernidad y el progreso tecnológico (Shrestha 1995; Fujikura 2001), situado en las ciudades y el extranjero (Pigg 1992; Shrestha 1995; Skinner y Holland 1996; Gellner 2015). Además, tiene un fuerte componente educativo y de género (Tamang 2000; Robinson-Pant 2001), siendo las mujeres uno de los principales objetivos a modernizar educando (Unesco 2000; World Economic Forum 2017).

La priorización de formas de vida urbanas y el acceso a trabajos asalariados a través de la escolarización entendidos, siguiendo a Appadurai (1996), como parte de una mega retórica de la modernidad y el desarrollo, han configurado un régimen de movilidad 
particular en Nepal, que exploramos a lo largo del artículo, contribuyendo empíricamente a la propuesta teórica de Glick Schiller y Salazar (2013). Para hacerlo, utilizamos una tercera y menos explorada aproximación al retorno, desde una perspectiva emic ${ }^{2}$ (Headland, Pike y Harris 1990; Díaz de Rada 2010), entendida como la percepción del proceso y las formas particulares de atribuirle significados por parte de sus actores. Lejos de reforzar las dicotomías sobre la positividad/negatividad del nexo movilidad-desarrollo, abordamos el retorno como experiencia narrada para explorar no tanto su impacto sino el proceso de una joven sherpa que vuelve al pueblo donde nació después de haber crecido y estudiado en India. El relato de Mingma permite acercarnos al retorno como experiencia situada y reflexiva que se (trans) forma en el proceso de su desarrollo.

El texto se organiza de la siguiente manera: primeramente, se sitúa el fenómeno de la movilidad y el desarrollo en Nepal desde una perspectiva de género; a continuación, se detalla la metodología y se presenta el caso de Mingma y su trayectoria de movilidad, que se discute posteriormente a la luz de los ejes de análisis derivados del marco teórico.

\section{MOVILIDAD, EDUCACIÓN Y GÉNERO EN NEPAL: CREENCIAS Y TENDENCIAS}

Nepal es un ejemplo perfecto de la vinculación positiva de la movilidad y el desarrollo, siguiendo la tendencia hegemónica de la modernidad que sitúa el desarrollo económico y tecnológico como impacto potencial y deseado de las migraciones. A nivel institucional, la migración ${ }^{3}$ es vista como un beneficio para el desarrollo del país, el crecimiento del PIB y la reducción de la pobreza a través de las remesas

2 Esta perspectiva es útil aquí porque nos permite «distinguir con la mayor precisión posible el plano de nuestras acciones, reflexiones e interpretaciones como investigadores, del plano de las acciones, reflexiones e interpretaciones de las personas cuyo comportamiento tomamos por objeto de análisis» (Díaz de Rada 2010: 75). King y Christou (2011), destacan la importancia de explorar esta perspectiva desde las creencias y ontologías del retorno de las personas migrantes.

3 Aunque este artículo se sitúa en un enfoque desde los regímenes de movilidad más que las migraciones, que entendemos como expresiones concretas de la movilidad humana, la mayor parte de la literatura específica en $\mathrm{Ne}$ pal está situada en este último paradigma.
(MOPE 2016). A nivel social, se asocia a un aumento del estatus personal y familiar (Kern y Müller-Böker 2015). La movilidad es un fenómeno masculinizado y con una sobrerrepresentación de la gente joven (MOPE 2016), para quien condensa un gran poder simbólico, siendo un ritual de paso moderno (Thieme y Wyss 2005; Kern y Müller-Böker 2015) o indicador de una madurez exitosa (Sijapati et al. 2017). En definitiva, la movilidad en Nepal ha sido promovida y celebrada hasta convertirse en algo que todo el mundo quiere (Thieme y Wyss 2005). El retorno es, en este contexto, un proceso clave no solo por el volver (en sentido geográfico) sino por las implicaciones económicas, sociales y simbólicas de su -supuesto- devolver (en sentido social). A las personas retornadas se les supone más entusiasmo en la inversión y emprendimiento (Sijapati et al. 2017) y la voluntad de hacer una contribución al lugar de origen (Agergaard y Broegger 2016). Tales devoluciones se deben entender en el contexto de las relaciones de parentesco, ya que los proyectos de movilidad tienen una marcada dimensión familiar en el contexto asiático (Piper 2008).

Aunque el patrón de movilidad dominante en Nepal es el motivado por el trabajo (Thieme y Wyss 2005; Sharma 2009; Kern y Müller-Böker 2015; Sijapati et al. 2017), en este artículo nos interesamos por las experiencias de movilidad vinculadas a la educación, intersección mucho menos explorada. La educación ${ }^{4}$ es, sin duda, un elemento central en los discursos hegemónicos del desarrollo (Shrestha 1995; Fujikura 2001; Robinson-Pant 2001; Caddell 2007), entendida como herramienta privilegiada para salir de las condiciones de subsistencia vinculadas a entornos rurales. Con el conocimiento escolar como base, se distingue a las personas abikasit (no-desarrolladas) de las bikasit (desarrolladas), que se vinculan a la modernidad, la vida en la ciudad y unas pautas particulares de consumo (Pigg 1992; Shrestha 1995; Levinson y Holland 1996; Skinner y Holland 1996). En relación al género, los discursos del desarrollo en Nepal han pasado del énfasis en el rol reproductivo de las mujeres a la introducción del mainstreaming y las perspectivas del empoderamiento (Davis 2015). Aun así, la mayoría de sus concreciones siguen teniendo un

4 Educación que se entiende, en general, en su significado restringido, como la participación en instituciones educativas formales, sean públicas o privadas, inspiradas y basadas en modelos europeos en cuanto a la organización del tiempo, los espacios y conocimientos (Harber 2014). 
enfoque economicista y de la eficiencia (Sibbons 1999; Bhadra 2001), en el que la educación para las mujeres es valorada por su potencialidad para generar otros cambios, como mejorar la calidad de vida de sus hijos o estimular el crecimiento económico (Kabeer 2015). La visión hegemónica de la mujer del tercer mundo que el desarrollo proyecta ${ }^{5}$ (Mohanty 1984; MacDonald 2016), también en Nepal (Sibbons 1999; Tamang 2000), contrasta con la heterogeneidad étnica y religiosa de las mujeres del país (Korzenevica 2016) y, por lo tanto, con la diversidad de formas de organizar los regímenes y relaciones de género. Los ideales modernos de familia se tornan hegemónicos y se consolidan tanto en las políticas públicas como en las prácticas del desarrollo, chocando con las comunidades que históricamente han tenido roles de género menos dicotómicos (Tamang 2000).

Estas configuraciones sociales y simbólicas generan unos flujos de movilidad específicos, que tienden a ir de lo rural a lo urbano y de dentro a fuera de Nepal. Tradicionalmente, la educación se ha considerado un impacto de la movilidad laboral (Thieme y Wyss 2005; Rao 2010), pero cada vez más es vista como motivación principal, como han evidenciado los enfoques de la fuga de cerebros y promocionan los discursos hegemónicos del desarrollo.

Nepal es un contexto tan relevante como complejo para el estudio de la movilidad educativa. En primer lugar, el acceso a la escolarización está condicionada por la movilidad geográfica hacia las zonas urbanas (Valentin 2015), hasta el punto de ser una de las principales causas de la despoblación rural (Childs et al. 2014). Otro tipo de movilidad relevante es la diaria, que muchos niños y niñas practican para llegar a la escuela, especialmente en las zonas rurales (Lind y Agergaard 2010). En tercer lugar, es importante en las decisiones educativas de los jóvenes varones, como una forma de ganar experiencias instrumentales y como un proceso de aprendizaje en sí mismo (Valentin 2012).

5 El desarrollo entendido como progreso lleva implícitas unas transformaciones específicas para las mujeres, que se proyectan de una forma homogénea como analfabetas y víctimas de unos sistemas culturales opresivos (RobinsonPant 2000; Tamang 2000), en el sí de una tendencia hegemónica a una conceptualización estática, homogénea y dicotómica de la mujer del tercer mundo (Mohanty 1984; MacDonald 2016).
El género, aunque pocas veces recibe una atención explícita en las investigaciones ${ }^{6}$ (Rao 2010), es un factor estructurante de la movilidad (Mora 2008; Piper 2008; Oso y Ribas-Mateos 2012; Cortés 2015; Cortés y Oso 2017). En Nepal, la presencia de las mujeres en los estudios de movilidad es ambivalente. Por lo general, son conceptualizadas como left behind o privadas de oportunidades de movilidad (Thieme y Wyss 2005; Maharjan, Bauer y Knerr 2012; Valentin 2015; Sijapati et al. 2017). El análisis de impacto se hace en tanto que mujeres de que se han quedado en el lugar de origen y destaca efectos positivos en la toma de decisiones pero también un aumento en sus cargas de trabajo (Maharjan et al. 2012). La feminización generalizada de las migraciones en los '90 (Mora 2008; Piper 2008; Vega 2016) supone romper con el estereotipo de la mujer reagrupada (Oso y Ribas-Mateos 2012) ${ }^{7}$. El patrón migratorio nepalí también se transforma: las mujeres empiezan a migrar en solitario y pasan a ser actores económicos en los procesos de movilidad (Bhadra 2007; Hamal Gurung 2015). Se ha señalado una actitud diferenciada de las mujeres a la hora de remitir: más predisposición, una motivación más altruista (Bhadra 2007) y como acto filantrópico para contribuir al desarrollo de la comunidad, siendo este último un fenómeno nuevo (Hamal Gurung 2015). La oportunidad para cambiar los roles de género (Cortés 2013), ganar libertades (Piper 2008), aumentar la autoestima y el estatus (Bhadra 2007; Hamal Gurung 2015) y la participación en actividades transnacionales (Levitt, DeWind y Vertovec 2006) son algunos de los impactos específicos de la movilidad con respecto al género bien establecidos en la investigación. Por otro lado, también existe la tendencia a reproducir los roles de género hegemónicos, tanto en los trabajos que las mujeres migrantes realizan (doméstico, cuidados) como en las relaciones transnacionales que se generan, siendo las mujeres las principales responsables del mantenimiento del cuidado y las comunicaciones (Baldassar 2008). El género es también relevante en Nepal con relación al tipo de movilidad. Debido a la mayor dificultad de las mujeres para conseguir edu-

6 En Nepal, los pocos análisis específicos sobre género se centran en la construcción y performatividad de las masculinidades (Sharma 2009; Maycock 2017).

7 A pesar de ello, no se puede negar que la visibilidad de las mujeres migrantes es todavía escasa, debido a que se da en y a través de circuitos informales en más medida que la migración masculina. Para un análisis detallado de la feminización de las migraciones en Asia ver Piper (2008). 
cación a través de las migraciones laborales «muchas ven la educación (y la alfabetización) como el fundamento del desarrollo personal y la movilidad social» (Pigg 1996; Ahearn 2001; LeVine 2006; Valentin 2014; citadas en Korzenevica 2016: 9). Como consecuencia, la tendencia entre las chicas es permanecer en la escuela (Sijapati et al. 2017) y viajar al extranjero para estudiar (MOPE 2016), mientras que los hombres ven en la movilidad laboral una inversión más segura. Asimismo, existe una correspondencia con el tipo de retorno que se proyecta para ellas, igualmente circunscrito en el ámbito educativo. La imagen de la mujer maestra tiene un fuerte poder simbólico en Nepal: la investigación ha enfatizado en numerosas ocasiones el efecto positivo de esta figura modelo para proveer confianza a las chicas y sus familias (Bista 2004) y sigue siendo promocionada para conseguir los tan recomendados niveles de paridad (Ministry of Education, Unicef y Unesco 2016). Finalmente, el retorno femenino se vincula con la transmisión de un mensaje específico sobre nociones alternativas de la igualdad de género y el empoderamiento (Agergaard y Broegger 2016).

Por lo tanto es crucial introducir la perspectiva de género en el análisis de los flujos de movilidad-inmovilidad y «explorar cómo se relacionan la movilidad geográfica y la movilidad social de los migrantes cruzada con el orden de género» (Oso, Sáiz-López y Cortés 2017: 300). Este articulo presenta un caso de movilidad educativa que, si bien es relevante porque supone llenar un vacío en la literatura, lo es aún más desde una perspectiva de género, sabiendo que existe una tendencia a la feminización de este tipo de trayectorias en Nepal. Además, alejándonos de los enfoques economicistas dominantes, exploramos la experiencia de retorno en sí misma, contribuyendo a llenar el vacío histórico en el estudio de las dimensiones sociales y culturales de las movilidades de retorno (Sharma 2009; Rao 2010; Cortés 2013; Agergaard y Broegger 2016).

\section{METODOLOGÍA}

Este artículo forma parte de una investigación etnográfica multisituada (Marcus 1995) iniciada en 2012 que tiene como punto de partida un estudio de caso en Gaun, una pequeña población en el valle de Solu-Khumbu (Himalaya Nepalés) y se extiende más tarde a la capital: Katmandú. Nos planteamos la investigación de las formas y modelos de T/A cultural en contextos formales y no formales (Castellsagué y Carrasco 2020a), el impacto de la escolarización y el desarrollo en la producción y reproducción de los regímenes de género (Connell 1990) y el análisis de las narrativas de las mujeres de Gaun y Katmandú (Castellsagué y Carrasco 2020b). Las mujeres sherpa (etnia mayoritaria en Gaun) atribuyen una gran importancia al trabajo: doméstico, organización de las cosechas, ceremonias y rituales. Por lo general, también administran los recursos económicos y materiales de la familia y tienen unas sólidas redes de apoyo mutuo femenino. La población de Gaun es mayormente femenina, debido a las largas ausencias de los hombres por motivos laborales. El transcurso de la investigación permite identificar la movilidad como un fenómeno relevante, registrando una gran cantidad de movimientos de la población: reales, narrados e imaginados. Siendo una característica idiosincrática en las zonas de montaña en Nepal y del pueblo sherpa, la movilidad diaria o estacional no es una novedad que se pueda atribuir a la modernidad. En cambio, otros tipos de movilidad hacia las ciudades y el extranjero han aumentado en los últimos años, provocando un importante descenso de la población de Gaun.

La trayectoria de Mingma se selecciona, de entre un extenso conocimiento etnográfico y las narrativas femeninas exploradas, como un caso singular que permite abordar en profundidad la intersección entre la movilidad, la educación y el desarrollo desde una perspectiva de género. Esto implica tener en cuenta aquellos aspectos sociales y relacionales de la movilidad y trascender los análisis estrictamente económicos que reducen la movilidad y el desarrollo a una cuestión de remesas cuantificables (Cortés 2013). Además, una lógica de investigación holística posibilita incluir la dimensión familiar de las migraciones, particularmente relevante en el contexto asiático ( $\mathrm{Pi}$ per 2008). Conocí a Mingma al poco tiempo de su llegada a Gaun, mientras visitaba la escuela donde ella trabajaba. Mi posición de maestra voluntaria en la escuela fue clave en nuestra relación, que se fue construyendo a partir de ese momento. Por un lado, nuestras diferencias eran evidentes, pero sentirnos ambas recién llegadas y ser mujeres en un entorno bastante masculinizado como es el personal docente de la escuela, ayudó a nuestro acercamiento. Además, el hecho de que ella me identificara como alguien que había venido a colaborar con la mejora de la educación se adaptaba perfectamente con sus expectativas de retorno al pueblo, como observaremos 
a lo largo del artículo. Las conversaciones empezaron en diferentes contextos del campo: recogiendo patatas en los campos de su familia durante la cosecha, tomando té en el descanso de la escuela, o simplemente caminando juntas en su barrio de Katmandú. Con el tiempo, fuimos avanzando hacia aproximaciones más formales de entrevista para la exploración de sus relatos (junto con otras informantes) con relación a la educación y el desarrollo (Castellsagué y Carrasco 2020b) y finalmente la reconstrucción de su historia de vida y, en particular, de sus experiencias escolares y de movilidad. En este artículo nos centramos en el análisis de su narrativa de movilidad con la intención de identificar temas emergentes y capturar las vivencias de una experiencia de retorno, en tanto que subjetividad travesada por ejes de identificación y desigualdad diversos y cambiantes (YuvalDavis 2006). Entendiendo las narrativas como un fenómeno fronterizo (Langellier 1989), las tomamos como un tipo de composición del discurso imbricado en fenómenos sociales en curso, en este caso los procesos de movilidad. A través de la narrativa de Mingma nos proponemos analizar el fenómeno del retorno mucho más allá de un movimiento físico, considerando las variables materiales, simbólicas e identitarias que conforman su complejidad.

\section{MINGMA Y SU TRAYECTORIA DE MOVILIDAD}

Me llamo Mingma Sherpa y tengo 23 años. Nací en Solu-Khumbu y cuando era pequeña me fui a Sikkim (India). Viví allí hasta los 21 años. Después volví a Solu-Khumbu por tres años, donde trabajé como profesora en una escuela -bueno... idos!- de educación secundaria. Allá fue cuando nos conocimos. Hasta que vine a Katmandú para encontrar un trabajo mejor, hace menos de un año ${ }^{8}$.

La vida de Mingma está indudablemente marcada por la movilidad. Aunque nació en Gaun, sus primeros recuerdos son en Sikkim $^{9}$, donde creció y se for-

8 Todas las citas textuales de este artículo corresponden a las diferentes entrevistas y encuentros con Mingma realizados a lo largo del año 2015 en Katmandú.

9 Pequeño estado del noroeste de India próximo a la frontera con Nepal. Alrededor del $70 \%$ de sus residentes son de origen nepalí, su idioma es lengua oficial y es un estado de tradición budista. Todo ello lo hace un destino atractivo para la población sherpa, como la familia de Mingma y muchas otras en Gaun, por su entorno culturalmente afín. mó. La vuelta a Gaun ${ }^{10}$ a sus 21 años, es para Mingma la vuelta "al pueblo», un lugar que formaba parte de su imaginario por todo «lo que le habían contado».

La vida en Gaun era buena. Me levantaba a las 7 $h$, me aseaba, tomaba té y preparaba la comida con mi hermana. Después del desayuno íbamos a la escuela. Pasábamos el día allá y después por la tarde tomábamos más té. Por la noche yo cocinaba y luego comíamos y a dormir. Cuando había festivales también podíamos asistir, es maravilloso.

Después de casi tres años en Gaun trabajando como maestra, Mingma decide establecerse en Katmandú, donde trabaja como telefonista.

Vine aquí para encontrar un trabajo mejor [...] Katmandú es maravilloso, aunque hay mucha contaminación y mucho ruido, pero exceptuando eso, está muy bien. Porque aquí hay un montón de opciones, si las podemos hacer. Estar en Katmandú está bien, porque está lejos del pueblo. Aunque también echo de menos a mis alumnos, y al resto de profesores.

Solemos encontrarnos en el barrio de Boudhanath, normalmente antes o después de que Mingma vaya a trabajar. Bajo del autobús justo delante de la entrada principal del templo y espero su llegada sentada en las escaleras de una tienda de souvenirs. La calle es una de las más atareadas de la ciudad, por lo que siempre hay mucho polvo y ruido. Aun así, la plaza redonda donde se encuentra el templo de Boudhanath es un pequeño oasis de tranquilidad. Al encontrarnos caminamos alrededor de la stupa y después nos adentramos en un callejón para tomar un té al estilo sherpa, nada fácil de encontrar en la ciudad. "Como en el pueblo ¿te acuerdas?», dice Mingma. "Aquí son familia, conocidos del pueblo, vamos a estar bien». Sentadas en una mesa, pedimos nun chia duita -dos té con leche, sal y mantequilla- y seguimos nuestra conversación.

\subsection{El RetOrno de Mingma: PREDOMINANCIA DE LAS DIMENSIONES FAMILIAR Y SIMBÓLICA}

Uno de los primeros temas que emerge en la reconstrucción de la trayectoria de movilidad de Ming-

10 El modo principal de subsistencia en Gaun es la agricultura, la ganadería a nivel doméstico y pequeñas actividades comerciales, normalmente puntuales e intermitentes. 
ma es la marcada dimensión familiar en la toma de decisiones del proceso de retorno.

¿Volver? Fue una decisión de mis padres, no mía. Mis padres insistieron en volver a Solu. Yo quería seguir estudiando allá [en Sikkim], vivir allá, quería... no quería venir a Nepal. En la televisión siempre decían Nepal es así, asá... nunca pensé que quisiera volver para ver cómo era. Pero tuve que hacerlo. Ellos... lo hicieron por mí, se preocupan por mí. Insistieron en que yo fuera al pueblo, pero les dije:

- ¡Nooo! Yo no quiero ir allá, quiero estudiar aquí, trabajaré aquí.

Pero dijeron:

- No, nosotros somos de Solu-Khumbu y tú tienes que hacer algo por tu pueblo. Nosotros no tenemos estudios y no podemos hacer nada, por eso tú tienes que hacerlo. Nos tienes que hacer sentir orgullosos.

Y dije:

- Okey, iré por vosotros.

Y así es que vine a Solu-Khumbu, y trabajé.

Mingma narra un doble posicionamiento. Desde un enfoque individual, reconoce que no quiere volver a Gaun, ya que sus proyecciones vitales y laborales son en Sikkim, pero desde una óptica familiar entiende las expectativas que hay sobre ella y su rol en el proyecto de movilidad y accede a desarrollarlo. Vemos la negociación de los planos individual y familiar que encarna Mingma como joven que retorna y que constituye una experiencia única de retorno más allá de la de su familia (Veale y Dona 2014). Por lo tanto, el retorno no solamente se ve plasmado como un movimiento físico, sino también simbólico, ya que Mingma no solo vuelve al pueblo donde nació, sino que (de)vuelve a su familia el esfuerzo que hizo para proporcionarle una educación en Sikkim.

Gracias a ellos [padre y madre] nosotros fuimos a Sikkim y hemos podido estudiar. Mi padre ha hecho mucho por nosotros, por eso vine a Solu-Khumbu, porque ahora nos toca a nosotros hacer algo por ellos.

Como señalan King y Christou (2011), el retorno es un proceso que activa relaciones de diversas naturalezas (económica, afectiva, simbólica), a menudo situadas en un marco de principios de reciprocidad y solidaridad. En este caso, la obligación de (de)volver que Mingma siente hacia su familia se sustenta en un marco de relaciones de parentesco basadas no solamente en los lazos afectivos sino también en unos determinados intereses económicos, sociales y sim- bólicos, que cobran sentido en los espacios transnacionales de su proyecto de movilidad, como veremos.

Las palabras de Mingma también reflejan las expectativas de otros actores sociales con relación al retorno, que claramente lo fomentan y reproducen la vinculación retorno-desarrollo explorado en la primera sección. Si bien asumen un impacto positivo de sus potenciales remesas en forma de trabajo, también las sitúan en el entorno escolar, como maestra. Tales expectativas no solamente recaen en Mingma sino también en sus hermanos menores:

El caso de mi hermana es similar al mío. Dorjee [el responsable de la escuela en Gaun], sabes? Llamó a mi padre y le dijo: «Me han dicho que tienes otra hija, nos gustaría que viniera a trabajar en la escuela también».

Sin embargo, lo hacen de una forma específica en relación al género. Vemos que las expectativas hacia la hermana de Mingma son similares a las suyas y la devolución esperada en el retorno se proyecta en forma de contribución escolar. Para el hermano de Mingma, en cambio, el retorno esperado es en forma de contribución en el negocio familiar en Katmandú. Si bien es cierto que él también trabaja durante un tiempo como profesor en un pueblo cercano, esto es percibido por su familia como una estrategia instrumental y temporal, mientras que se espera de él la participación en otro tipo de trabajos, como el trekking o la gestión de restaurantes. Estas diferenciaciones se corresponden con las dinámicas de movilidad y retorno generizadas señaladas en la primera sección, que nos sirven como marco socio-histórico para contextualizar los regímenes de movilidad en los que se sitúa la experiencia de Mingma.

Aunque la dimensión familiar de la movilidad ha sido anteriormente enfatizada en Nepal con respecto al trabajo (Regmi y Tisdell 2002; Thieme y Wyss 2005; Poertner, Junginger y Müller-Böker 2011 ), se tiende a conceptualizar la migración educativa como un proyecto más personal de la juventud Nepalí. El caso de Mingma, en cambio, muestra que la estrategia educativa también responde al patrón inversión-retornodevolución de los enfoques laborales y la lógica migratoria familiar.

En primer lugar, por el planteamiento del proyecto de movilidad, concebido desde un inicio con una intencionalidad a largo plazo.

Mis padres no tienen educación, fue por eso que se mudaron a Sikkim. Lo hicieron por la educación, 
porque mi padre no fue a la escuela y pensaba: «si me quedo aquí [en Solu-Khumbu], mis hijos serán como yo, y no lo quiero. Así que me iré a algún lugar, trabajaré y enseñaré a mis hijos». Así que gracias a ellos nosotros fuimos a Sikkim y hemos podido estudiar.

El primer flujo (Gaun-Sikkim) es visto como una apuesta educativa por parte de los progenitores con sentido a largo plazo, ya que se completa volviendo a Gaun para contribuir al desarrollo del pueblo (Figura 1). Por lo tanto, siendo la segunda generación la que completa el ciclo del proyecto de movilidad familiar, identificamos el carácter profundamente intergeneracional del proyecto de movilidad.

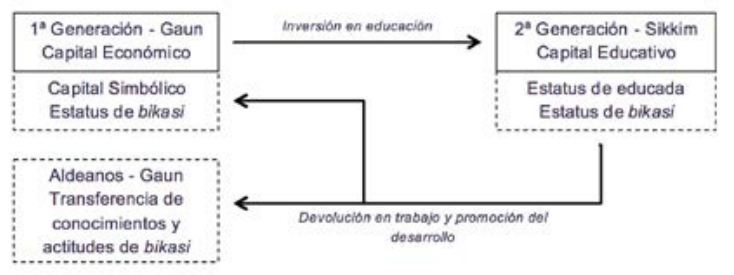

FIGURA 1.-Flujos de inversión y retorno en la movilidad como proceso intergeneracional. Elaboración propia.

A lo largo de las siguientes secciones se explora en profundidad las formas en las que se dan tales flujos de retorno. Si bien Mingma nos habla del retorno imaginado, tampoco ve del todo claro volver: en general porque considera que está bien en Sikkim y no ve necesidad de cambiar y, específicamente, por «lo que le han contado» del pueblo, que no necesariamente son cosas que considere positivas. Contrariamente a la tendencia investigada sobre el mito del retorno (Madawi 1994; King 2000; Bolognani 2007), vemos como la evocación al lugar de origen no siempre incentiva el deseo de volver, sino que puede también generar escepticismos. A continuación, exploramos las complejidades del retorno vivido de Mingma que le plantea retos y contradicciones, analizando con detalle cuales son las encrucijadas sociales e identitarias en las que se encuentra en y a través la experiencia de (de)volver a Gaun.

\subsection{NEGOCIACIONES DE LA IDENTIDAD Y SITUACIÓN EN EL MAPA SOCIAL}

Cuando conocí a Mingma manifestaba encontrarse en un momento reflexivo respecto a su identidad. Se sentía parte del pueblo porque su familia es de allá, pero era como si ella no perteneciera más a ese lugar. A través de las conversaciones compartidas me di cuenta que la experiencia de movilidad de Mingma la había transformado al menos, en dos sentidos. En primer lugar, el nivel educativo adquirido la había posicionado como una mujer educada, estatus que le debía permitir tomar un rol de trabajo particular: el de profesora. De hecho, Mingma no tiene una formación específica para este trabajo, aunque el hecho de haber sido una buena estudiante en Sikkim la convierte en una buena candidata. Pero la movilidad también está simbólicamente asociada al desarrollo y el haber vivido en el extranjero la convierte en una bikasi, persona desarrollada. A través de su narrativa descubrimos como su nueva posicionalidad -no siempre consciente o deseada- se construye a través de una negociación de mensajes, valores, expectativas y autoridad entre ella y los otros.

Mingma se refiere a la gente de Gaun en tercera persona y expresa una visión particular sobre «ellos», sus dinámicas y actitudes. "Ellos» constituye una de las razones por las cuales Mingma «lo pasó mal» en su llegada al pueblo:

Por los aldeanos ${ }^{11}$, porque ellos son un poco... crean situaciones muy malas, y a veces cuestan de tratar. Siempre están creando problemas y chinchando. También tienen malos hábitos, por ejemplo, después de tomar alcohol pelean por cualquier cosa.

Mingma hace hincapié en las actitudes para calificar como problemáticos ciertos hábitos de las personas de Gaun. De la misma forma que establece una distinción hacia los otros, justifica su visión como parte de un nosotros, formado por la gente que ya no vive en el pueblo y con la cual se identifica. De esta forma, apoya su percepción con la de otras que «piensan lo mismo»:

\begin{abstract}
La mayoría de veces cuando la gente de Katmandú va al pueblo, todos comentan: "Míralos, no saben cómo vestir, no saben cómo comer, no saben cómo hacer las cosas». [...] Porque ellos están en la ciudad y tienen un buen entorno, pero en el pueblo es imposible. Siempre comentan cosas malas.
\end{abstract}

Mingma construye ambas realidades como opuestas y sitúa la aprobación o desaprobación de cierto

11 Mingma utiliza el término villager, que hemos traducido como aldeano para mantener su carga simbólica, ya que se utiliza en Nepal como categoría de distinción social para identificarse como bikasi, «desarrollado» (Pigg 1992). 
estilo de vida en ese paradigma dicotómico. La imagen del otro como «aldeano» está influenciada por una ideología de la modernidad y el bikas (ver Pigg 1992): ciertos códigos de vestir y comer, la posesión de riqueza y conocimiento escolar. No solo se atribuyen ciertos hábitos como tradicionales en oposición a la modernidad, sino que se califican como problemáticos. Este caso añade la movilidad como elemento central en tal distinción, asociando el bikas con aquella que ocurre en una dirección particular: hacia Katmandú o el extranjero. Mingma se sitúa en un nosotros construido alrededor de lo urbano, moderno, más desarrollado. Si bien sabemos que el retorno está directamente relacionado con las narrativas de pertenencia a diferentes niveles (familiar, local, nacional), el caso de Mingma nos revela la complejidad en el caso de las segundas generaciones en el retorno. Al inicio de su relato Mingma argumenta su intención de (de)volver a Gaun por una vinculación familiar y local (somos del pueblo). Sin embargo, a lo largo del proceso, su experiencia la sitúa en un marco identitario que va más allá de Gaun como espacio físico, y trasciende al plano simbólico en una nueva identificación colectiva (las personas del pueblo que ya no viven allá, que son bikasi). Por todo ello, no puede entenderse tal posicionamiento como una tendencia individual a sentirse superior sino como producto estructural de los significados sociales del desarrollo (Pigg 1993).

Otro de los aspectos que identificamos en la narrativa de Mingma es la transferencia de cierta responsabilidad a la gente del pueblo por «no saber» como hacer las cosas "apropiadamente». Atribuye esta situación a las condiciones materiales y a «no haber tenido educación», especialmente las mujeres. Por lo tanto, vemos como el conocimiento/experiencia escolar -en este caso su ausencia- constituye otro factor en la construcción del abikasit (no-desarrollado), de la misma forma que la oportunidad de llegar a ser desarrollado, se vincula a la movilidad hacia Katmandú:

En Katmandú [los aldeanos] pueden obtener una buena educación, y un buen sistema sanitario, y pueden aprender alguna cosa y desarrollar su personalidad. [...] En el pueblo no hay instalaciones para comprar ropa, siempre usan las manos para comer... en Katmandú utilizan cuchara y tenedor, es diferente.

Finalmente, de la narrativa de Mingma sobre los aldeanos emerge la noción de cambio:
Ellos deberían cambiar: la forma en la que visten, la forma en que hablan, la forma en la que comen [...] Pero poco a poco los aldeanos están aprendiendo estas cosas. Ahora han hecho un buen cambio.

Mingma conceptualiza la alteridad como gente necesitada de un cambio para avanzar en la escala del desarrollo. Un cambio hacia formas de vida más modernas y educadas que la gente de Gaun necesita aprender. Como exploramos en la siguiente sección, esta noción es central en la retórica del retorno, que sitúa a las personas que han tenido experiencias particulares de movilidad como agentes y promotores de dicho cambio.

\subsection{Mingma COMO Agente DE DESARROLlo}

El objetivo del retorno de Mingma, sus expectativas y el papel que aspira a desempeñar en el pueblo se circunscriben en la mencionada retórica del retorno: se espera que jóvenes que han obtenido una mejor educación en el extranjero, al volver, (re)inviertan una parte del capital adquirido (Agergaard y Broegger 2016) y contribuyan así al desarrollo de las zonas rurales. En este caso particular, en forma de transferencia de conocimientos y actitudes (Figura 1).

Mingma atribuye a su llegada ciertos cambios en la gente, especialmente las chicas jóvenes que se identifican con ella:

\footnotetext{
Sí, estuvimos en India y por eso llevamos ese tipo de ropa: tejanos, camisetas. Después los aldeanos también se inspiraron: "Mira, ella se viste así, yo también lo voy a comprar». "Ellos [la familia de Mingma] se han desarrollado un montón", todos lo dicen en el pueblo. "Han cambiado mucho". Las chicas siempre dicen «Oh, Dios. Si tú no hubieras llegado, no habríamos cambiado así».
}

La ropa y el aspecto físico son uno de los cambios más evidentes en el caso del retorno femenino (Levitt 1998; Agergaard y Broegger 2016). Mingma entiende la imitación de su forma de vestir como un indicador de cambio positivo. En segundo lugar, Mingma señala transformaciones en el tipo de relaciones entre la gente del pueblo:

Muchas de las ceremonias y festivales, ellos no solían ayudarse los unos a los otros, eso he escuchado. Antes que nosotros llegáramos al pueblo, no se ayudaban.

En tercer lugar, cambios relevantes hacia las mujeres, que reproducen la conceptualización de mujer 
rural arquetípica, central en los discursos hegemónicos del desarrollo en Nepal (Pigg 1992):

Mingma: ¿Cambio? Sí, lo que cambiaría es su personalidad y forma de pensar. Si está bien, ellas creen que está mal. Tienes que hacerlo así. Y ellas dicen: «No, está mal, tienes que hacerlo asá». Ellas siempre dicen lo contrario. Así que lo más importante es cambiar sus concepciones, su forma de pensar.

E: Entonces ellas tendrían que pensar más como...

M: Tendrían que pensar más como mujeres educadas, tienen que estar educadas. Muchas de las mujeres piensan así porque ellas no tuvieron una buena educación.

Mingma identifica su función como agente de desarrollo en promover cambios hacia una nueva forma de vestir, relacionarse y pensar más moderna y educada (Figura 1). Este posicionamiento es doblemente relevante en Nepal: por la perspectiva de género y la educación, ya que los programas escolares suponen una buena oportunidad para las chicas de entrar a formar parte de las redes y la familia del desarrollo (Robinson-Pant 2001). La posición de Mingma refleja una paradoja con relación a los tipos de remesas que aporta a Gaun y el contexto donde se da la transferencia de conocimientos. Por un lado, la mayoría de discursos sobre el retorno sitúan los cambios sociales en los sistemas de prácticas (Levitt 1998) a través de su incidencia en el ámbito formal, la escuela en este caso. En cambio, Mingma hace más énfasis en los ámbitos informales, identificando cambios en las estructuras normativas (Levitt 1998) ${ }^{12}$. Es decir, el rol simbólico de maestra que le debería permitir transmitir conocimientos modernos tiene poco que ver con los mecanismos informales que su narrativa señala como efectivos para el cambio de facto. Por lo tanto, esta investigación muestra como la tendencia a situarse como agente femenino de desarrollo no solo se produce a través de las instituciones explícitamente relacionadas con el bikas (ONG y ONGI) sino también a través de las que se vinculan a él de una forma simbólica (la escuela) o simplemente fuera de ellas, a través de las relaciones inter-personales en ámbitos informales.

12 Entre los tipos de remesas sociales, las estructuras normativas corresponden al ámbito de las ideas, valores y creencias, mientras que los sistemas de prácticas hacen referencia a las acciones y prácticas concretas a nivel individual o en las organizaciones (Levitt 1998: 933-935).
El hecho de verse capaz de provocar cambios en otros y en su entorno proporciona a Mingma un sentido de agencia que la hace sentir muy valorada:

La gente del pueblo siempre dice: «Sí, es por ti que está pasando aquí [los cambios, el desarrollo]». Hace sentir como que... «oh, isoy algo!» Me hace sentir bien, porque cuando la gente me aprecia me siento bien. [...] Pero por otro lado yo siempre digo que no nos tienen que copiar. Somos diferentes, sois diferentes. No tenéis que copiarnos. Nosotros somos así, y vosotros tenéis que quedaros como estáis.

Si bien la movilidad como promotora de agencia ha sido especialmente enfatizada con respecto a las mujeres (Hamal Gurung 2015), la narrativa de Mingma problematiza esta vinculación positiva. En primer lugar, expresa contradicciones con relación al cambio social que se está produciendo en Gaun. Por otro lado, ejercer como agente de cambio es más difícil de lo esperado y Mingma no siempre puede cumplir con las expectativas. En la siguiente sección descubrimos el poco explorado otro lado del retorno, en el que Mingma muestra la complejidad de las experiencias vividas, donde las contradicciones y negociaciones son parte del proceso de (de)volver, en todas sus dimensiones.

\subsection{Problemas, Resistencias y la falacia del RETORNO}

Cuando conocí a Mingma, recién llegada a Gaun, expresaba las dificultades en las que se encontraba y como "echaba de menos Sikkim, quería volver a Sikkim». En primer lugar, debido a resistencias por parte de la gente de Gaun ante el cambio social.

Cuando intentamos decirles: «Esto está mal, lo tienes que hacer así», ellos dicen: "Oh, ¿ahora nos vas a enseñar? Yo soy mayor que tú, soy tu senior. ¿Cómo me vas a enseñar?» $Y$ eso es muy malo.

Mingma no comprende las resistencias al «buen» cambio frente a «malas» prácticas de la gente de Gaun y las atribuye a un sistema jerárquico particular: la edad, en que los mayores enseñan a los más jóvenes. Aunque factor poco explorado en tanto que eje generador y reproductor de estructuras de poder y legitimidad ${ }^{13}$, Mingma lo identifica como limitante en su capacidad de transformar la realidad local. En tan-

13 En Nepal, la mayoría de investigaciones sobre la edad se han producido alrededor de los procesos de envejecimien- 
to que las movilidades ocurren a través de cuerpos travesados por el género, la raza y la edad (Büscher y Urry 2009; Veale y Dona 2014), esta narrativa muestra como tales categorías se negocian en el retorno, siendo unas u otras más relevantes en función del contexto. Los datos etnográficos también muestran la edad como factor relevante en Gaun, por ejemplo, en la organización del trabajo y la transmisión del conocimiento. Aun así, las jerarquías en relación con la edad no se aplican a todos los contextos ya que la escuela -una institución dirigida por gente relativamente joven-, lejos de ser desafiada, es validada como la mejor opción para el desarrollo y la modernización. La explicación que proponemos tiene que ver con la paradoja sobre el grado de formalidad del contexto y su vinculación a la modernidad y el desarrollo. El hecho de que la gente de Gaun relacione la escuela con un conocimiento nuevo, externo haría más flexible el cambio en la forma y las jerarquías de transmisión del conocimiento. En el ámbito informal, en cambio, justamente donde Mingma aspira a incidir, hay más resistencias a los cambios sociales, ya que no están explícitamente asociados al conocimiento escolar. Por lo tanto, la legitimidad para producir cambios no está solamente relacionada con el estatus de bikasi sino en la relación de este con cada contexto particular.

En segundo lugar, Mingma destaca envidias hacia su familia, que apelan a los procesos de distinción social producidos a través de la movilidad y, en particular, al cambio de estatus que supone volver al pueblo con nuevos capitales adquiridos (Figura 1).

\begin{abstract}
Muchos de los aldeanos están celosos de nosotros. Todo el mundo dice: "Míralos, tienen tres hijos y todos están trabajando, tienen una buena educación y pueden hacer lo que sea. ¿Y nosotros? Nosotros no tenemos nada de eso» [...] [Los aldeanos] ven el ejemplo. Eso tiene cosas buenas también claro, pero de algún modo también es malo, porque alguna gente está celosa. Siempre dicen: «Tres hijos trabajando en el pueblo y ganando dinero, y la mamá gana dinero del hotel, y el papá se va de trekking. Todo el mundo está ganando dinero... ¿Dónde guardan tanto dinero?».
\end{abstract}

En tanto que la movilidad ha sido un proyecto familiar, el nuevo estatus no se atribuye solo a Mingma sino a toda su familia, resultado que apoya otras in-

to de la población y problemáticas como la marginalización y vulnerabilidad (e. g. Beall y Goldstein 1982; Parker 2012). vestigaciones sobre migraciones en Nepal (Agergaard y Broegger 2016). Sin embargo, también muestra cómo ocurre de forma similar con las envidias y celos, que se proyectan no solamente sobre ella sino hacia toda su familia. Esta aproximación está sin duda menos presente en la literatura. Así, el caso de Mingma y su familia pone de manifiesto el retorno como estrategia de obtención y acumulación de estatus, pero también como generadora de nuevas tensiones sociales, siempre contextualizadas en un marco complejo de obligaciones de parentesco. Tales procesos de consolidación y desafío de estructuras de poder y reconocimiento son clave en la comprensión y el análisis de los regímenes de movilidad.

Mingma narra cómo, ante las posiciones incómodas en las que se encuentra, decide modificar su conducta:

Al final yo desistí en seguir explicándoles. Dejé que hicieran lo que quisieran, que dijeran lo que quisieran. Vale, ellos escuchan y olvidan. No está bien discutir por tonterías y no está bien discutir con los borrachos. Lo que hay que hacer es olvidarlo.

Una de las consecuencias de un fracaso en el retorno es la (re)emigración (Sinatti 2011). La frustración de Mingma ante la incapacidad de cumplir su supuesto rol representa uno de los factores explicativos de su traslado a Katmandú, después de aproximadamente tres años en Gaun. En Nepal, y especialmente en la migración educativa, pocas personas retornan definitivamente para ocupar posiciones de más poder en los pueblos, como solía ocurrir en el pasado (Agergaard y Broegger 2016). Puede cuestionarse también la direccionalidad del proceso de retorno y las mismas categorías de origen y destino, al concebir las segundas generaciones como nuevas primeras generaciones de migrantes. Habiendo sido socializada en Sikkim, el retorno para Mingma puede tener características de una primera emigración con Sikkim como lugar de origen, generando vínculos y redes transnacionales reversos a los de sus padres (King y Christou 2011; Gil Araujo et al. 2013). A lo largo de su narrativa, hemos visto como Mingma va desmontando las ideas de su retorno imaginado y su experiencia se acerca más a un retorno provisional (Oxfeld y Long 2004), que le da la oportunidad de valorar la (im)posibilidad de un retorno definitivo a Gaun. De hecho, sus deseos para el futuro contemplan nuevos movimientos, llenos de retos y complejidades.

Yo he probado mi suerte aquí [en Katmandú], y conseguí un trabajo. Eso me hace sentir bien, que 
puedo hacerlo. Y tengo que hacerlo. [...] Me gustaría ir a Sikkim, ya lo veremos. Si encuentro un trabajo allá me podría quedar. Sikkim era muy tranquilo, había mucha paz, y era maravilloso y muy bonito. Y la gente es muy buena. Mi infancia fue allá, y ilo echo de menos! [...] Pero entonces sería lo mismo, tendría trabajo allá, pero me sentiría sola otra vez, porque ahora mi madre y mi padre están aquí, mi hermana y hermano están aquí. Y sola allá... no es fácil. Ellos siempre me dicen: «Te tienes que casar aquí, porque si te casas en Sikkim te tendrás que ir». Y cuando me vaya mis padres se preocuparán mucho por mí, siempre se preocupan por si estoy bien. Así, un poco de presión... así que es mejor que me case aquí, y me quede cerca de ellos. Nos podemos ver, nos podemos encontrar y yo los puedo cuidar. iVeremos lo que sucede!

También en sus expectativas, la narrativa de Mingma revela su conceptualización de cualquier estrategia de movilidad como un proyecto familiar, en el que incluye los deseos de sus padres, hermanos y hermanas. Por un lado, vemos como la marcada dimensión familiar del proyecto de movilidad genera que los flujos de inversión-devolución se sitúen en una lógica de obligaciones parentesco, así como los estatus adquiridos y las expectativas. Sin embargo, el tipo de negociaciones que emergen de estos nuevos estatus y roles no son homogéneos, sino que se encuentran travesados por otros marcadores personales de posición e identidad en cada contexto. Así, las jóvenes que participan de los proyectos de movilidad encarnan experiencias singulares que no pueden quedar invisibilizadas tras las de sus familias (Veale y Dona 2014). La dimensión del género vuelve a ser relevante aquí para significar la responsabilidad que siente Mingma hacia del mantenimiento de las relaciones familiares y su cuidado. El género, tal como apunta Baldassar (2008), media no solamente los proyectos de movilidad de la joven sino también sus expectativas laborales y vitales en el futuro.

\section{LAS CONTRADICCIONES DEL DESARROLLO Y LA RETÓRICA DEL RETORNO EN NEPAL}

La movilidad educativa en Nepal aparece representada de una forma dual. Mientras la movilidad diaria y en contextos rurales supone una barrera a la escolarización (Levine 2006; Ministry of Education, Unicef y Unesco 2016), se vincula a la exclusión social, el subdesarrollo y se identifica como un poverty trap (Lind y Agergaard 2010); la movilidad a los centros urbanos/extranjero es vista como el acceso simbólico a la movilidad social (Lind y Agergaard 2010). En tanto que se le supone a la movilidad un impacto opuesto en función de qué dirección tome, quién la practique o su escala geográfica, identificamos regímenes particulares de movilidad (Glick Schiller y Salazar 2013) imbricados en la estratificación social y cultural de Nepal. En este contexto, la importancia simbólica del retorno radica en la capacidad del que (de)vuelve de desarrollar su lugar de origen. Sin embargo, nuestra investigación: 1) presenta el retorno como un fenómeno polisémico, 2) muestra la existencia de una retórica del retorno en los discursos del desarrollo que está, además, muy vinculada a la educación y 3) problematiza dicha retórica desde la perspectiva de género a través del relato sobre la experiencia de Mingma. Con ello, contribuimos a mostrar empíricamente una más de las contradicciones del desarrollo en Nepal.

En primer lugar, hemos visto como las distinciones sociales vinculadas a los procesos de movilidad están imbricadas en paradigmas más amplios del desarrollo y la educación, en los que haber estudiado en el extranjero sitúa a Mingma en un nuevo estatus social de persona educada y desarrollada. Como hemos podido ver a través de la imagen que Mingma tiene de las otras mujeres de Gaun, la vinculación entre la movilidad y el desarrollo es particularmente relevante para las mujeres, que se proyectan como más necesitadas de cambios hacia la modernidad y la educación (Mohanty 1984).

En tanto que la construcción del otro se hace en base a la movilidad y el conocimiento, proponemos que la configuración del régimen de movilidad (Glick Schiller y Salazar 2013) en Nepal se encuentra vinculada con un régimen de conocimiento particular. Esto es, se fomenta y celebra una movilidad hacia la modernidad que se asocia con un conocimiento escolar y académico; por encima de otros tipos de movilidad y conocimientos que se conceptualizan como tradicionales y rurales. En este contexto, hemos identificado la existencia de una retórica del retorno que reproduce las tendencias hegemónicas celebratorias de su impacto positivo, de forma específica con relación al género y el desarrollo en Nepal. El retorno de Mingma va más allá de una recuperación de capital humano, sino que es estructurado por $-\mathrm{y}$ a la vez estructurante en- las relaciones del desarrollo (bikasi-abikasi) y la educación (educado-no educado). En la línea de Cortés (2009 
y 2013), nuestros resultados contribuyen a mostrar la faceta política e ideológica del nexo movilidaddesarrollo, que facilitan y promueven ciertos tipos de movilidad, educación y desarrollo.

Esta investigación problematiza empíricamente la retórica del retorno, en tres sentidos. En primer lugar, las malas experiencias de Mingma ponen en entredicho la imagen de retorno ideal o imaginado (Oxfeld y Long 2004) y las investigaciones que solamente señalan el impacto positivo del retorno (Agergaard y Broegger 2016). En cambio, hemos evidenciado las negociaciones y contradicciones del proceso en el plano de las identidades, materialidades y posicionalidad ante el poder y la autoridad. De esta forma contribuimos a la difícil tarea reivindicada por Cortés de entender el nexo migración-desarrollo más allá del impacto del uno sobre el otro sino desde una perspectiva de la reciprocidad (2013: 131). Por otro lado, Mingma muestra las dificultades de transformar el capital adquirido en movilidad por educación en el contexto local, con respecto al género pero también la edad, explorando las relaciones entre varias formas de desigualdad horizontal (Kabeer 2015). Situar estos resultados en una perspectiva de los regímenes de movilidad (Glick Schiller y Salazar 2013) implica pensar en el retorno de Mingma como un proceso que consolida ciertas estructuras de poder -las del desarrollo y la educación escolar- a la vez que desafía otras -las de género y edad-. Estas contribuciones son especialmente relevantes porque sitúan los debates de la interseccionalidad en el desarrollo ${ }^{14}$ en un contexto etnográfico concreto y a través de una experiencia situada. Finalmente, hemos visto como las tipologías de retorno son categorías vivas, que se negocian y (trans)forman a partir de las experiencias vividas en el camino.

A pesar del foco cada vez más individual en las nuevas conceptualizaciones de la movilidad ${ }^{15}$, el caso de Mingma muestra su todavía relevante dimensión familiar, en que los movimientos están inscritos en estrategias más amplias de inversión y situados en una lógica de obligaciones de parentesco. La óptica generacional es una de las explicaciones de la poca presencia del mito del retorno en Mingma, que tiene

14 Ver, entre otras, las aportaciones de Yuval-Davis (2006 y 2016) o Nightingale (2011) para el caso de Nepal.

15 Los cambios tecnológicos refuerzan la idea de las personas como nodos móviles que conforman redes en movimiento (Sheller y Urry 2006). más características de primera generación de migrantes que de retornados, que sí cumplen sus progenitores. Esta investigación permite ver el avance conceptual que aporta la perspectiva de género en el análisis de las movilidades para trascender las perspectivas individuales y (re)conocer el todavía relevante papel de la familia y el entorno social en los procesos de retorno. Esto es, en definitiva, constatar el carácter profundamente social de las movilidades.

En el plano de la identidad, el retorno ha tenido una fuerte carga ideológica. Presupone una relación de pertenencia entre los individuos y sus lugares de origen (Pascual de Sans 1983) y es visto como una performance of belonging (Fortier 2000) o un regrounding (Ahmed et al. 2003) (en King y Christou 2011). Estas aproximaciones al retorno coinciden con la manera en que Mingma y su entorno construyen sus expectativas. Su experiencia, sin embargo, presenta el retorno como negociación y distinción, como se sitúa y es situada en el campo social en una posición ambivalente. Esta investigación nos permite cuestionar las bien establecidas categorías analíticas en el desarrollo: extranjero/local, cooperante/receptor de ayuda y demostrar que se encuentran en constante negociación interna y externa. El desarrollo, siguiendo a Pigg (1992), más que provocar choques entre sistemas culturales, produce «situaciones en las cuales las personas empiezan a verse de ciertas maneras» (en Escobar 1998: 93). Si bien la literatura ha descrito la situación del migrante como espacio medio entre mundos, tiempos y sociedades (Cavalcanti y Parella 2013; Hamal Gurung 2015; Kern y MüllerBöker 2015), nuestra investigación concluye en la necesidad de (re)pensarlos con relación al desarrollo (Shiva 1989; Robinson-Pant 2001; Gellner 2015). Proponemos que las movilidades y el retorno generan espacios físicos de negociación a la vez que sitúan a las personas -no solamente las móviles- entre los espacios sociales del desarrollo, generando distinciones sociales y simbólicas atravesadas a la vez por otros ejes de estratificación social como el género y la edad en el caso de Mingma. Esta idea de limbo o dualidad tan presente en la literatura sobre migraciones y desarrollo nos es útil aquí en su sentido de negociación y no de oposición. Lejos de entender la situación de Mingma entre dos mundos claramente separados, hemos querido remarcar su posición dialogante entre varias realidades que se retroalimentan en y a través de ella misma, sus experiencias y el simbolismo de lo que representa. 


\section{BIBLIOGRAFÍA CITADA}

Agergaard, Jytte y Ditte Broegger. 2016. «Returning Home: Migrant Connections and Visions for Local Development in Rural Nepal». Geografisk Tidsskrift - Danish Journal of Geography 116(1): 71-81. doi: <https://doi.org/10.1080/00167 223.2015.1118706>

Ali, Nazia y Andrew Holden. 2006. «Post-colonial Pakistani Mobilities: The Embodiment of the 'Myth of Return' in Tourism». Mobilities 1(2): 217-242. doi: <https://doi. org/10.1080/17450100600726605>.

Appadurai, Arjun. 1996. Modernity at Large: Cultural Dimensions of Globalization. Minneapolis: University of Minnesota Press.

Baldassar, Loretta. 2008. "Missing Kin and Longing to Be Together: Emotions and the Construction of Co-presence in Transnational Relationships». Journal of Intercultural Studies 29(3): 247-266. doi: <https://doi. org/10.1080/07256860802169196>.

Bereményi, Bálint Ábel y Sílvia Carrasco. 2017. «Bittersweet Success. The Impact of Academic Achievement Among the Spanish Roma after a Decade of Roma Inclusion», en W. T. Pink y G. W. Noblit (eds.), Second International Handbook of Urban Education., vol. 1: 1169-1198. Cham: Springer International Handbooks of Education. doi: <https://doi. org/10.1007/978-3-319-40317-5>.

Bhadra, Chandra. 2001. «Gender and Development: Global Debate on Nepal's Development Agenda». Contributions to Nepalese Studies 28(1): 95-107.

Bhadra, Chandra. 2007. «International Labor Migration of Nepalese Women: Impact of their Remittances on Poverty Reduction». Asia-Pacific Research and Training Network on Trade Working Paper Series n. 44.

Bista, Min Bahadur. 2004. «A Review of Research Literature on Girls' Education in Nepal». UNESCO Kathmandu Series of Monographs and Working Papers, vol. 3. Katmandú: United Nations Educational, Scientific and Cultural Organisation.

Bolognani, Marta. 2007. "The Myth of Return: Dismissal, Survival or Revival? A Bradford Example of Transnationalism as a Political Instrument». Journal of Ethnic and Migration Studies 33(1): 59-76. doi: <https://doi. org/10.1080/13691830601043497>.

Büscher, Monika y John Urry. 2009. «Mobile Methods and the Empirical». European Journal of Social Theory 12(1): 99-116. doi: <https://doi.org/10.1177/1368431008099642>.

Caddell, Martha. 2007. «Education and Change: A Historical Perspective on Schooling, Development and the Nepali Nation-State», en K. Kumar y J. Oesterheld (eds.), Education and Social Change in South Asia: 251-284. Nueva Delhi: Orient Longman.

Carling, Jørgen, Marta Bolognani, Marta Bivand, Rojan Tordhol, Ceri Oeppen, Erlend Paasche, Silie Vatne y Toye Heggli. 2015. Possibilities and Realities of Return Migration. Oslo: PRIO.
Castellsagué, Alba y Sílvia Carrasco. 2020a. «Development, Education and Gender: Challenging the Empowerment Rhetoric from an Ethnographic Study in Rural Nepal». Ethnography and Education. doi <10.1080/17457823.2020.1764 856>.

Castellsagué, Alba y Sílvia Carrasco. 2020b. «Schooling and Development. Global Discourses and Women's Narratives from Nepal». Compare: A Journal of Comparative and International Education. doi $<10.1080 / 03057925.2019 .17098$ 03>.

Cavalcanti, Leonardo y Sònia Parella. 2013. «El retorno desde una perspectiva transnacional». REMHU - Revista Interdisciplinar da Mobilidade Humana XXI(41): 9-20.

Childs, Geoff, Sienna Craig, Cynthia M. Beall y Buddha Basnyat. 2014. «Ethnically Tibetan Communities of Nepal Depopulating the Himalayan Highlands: Education and Outmigration from Ethnically Tibetan Communities of Nepal». Mountain Research and Development 34(2): 85-94.

Connell, R. W. 1990. "The State, Gender, and Sexual Politics». Theory and Society 19: 507-544.

Cortés, Almudena. 2009. "Los antecedentes políticos del codesarrollo: la reinvención del nexo entre la migración y el desarrollo en el sur de Europa», en Francisco Checa, Juan Carlos Checa y Ángeles Arjona (eds.), Las Migraciones en el Mundo, Desafíos y Esperanzas: 55-96. Barcelona: Icaria Antracyt.

Cortés, Almudena. 2013. «Gender, Andean Migration and Development: Analytical Challenges and Political Debates», en Laura Oso y Natalia Ribas-Mateos (eds.), The International Handbook on Gender, Migration and Transnationalism: 127144. Cheltenham: Edward Elgar Publishing. doi: <https:// doi.org/10.4337/9781781951477.00013>.

Cortés, Almudena. 2015. «Migración, género y estrategias intergeneracionales de movilidad social entre Ecuador y España», en Actas del VIII Congreso sobre Migraciones Internacionales en España (Granada,16-18 de septiembre 2015): S30/91-S30/100. Granada: Instituto de Migraciones.

Cortés, Almudena, Mercedes Fernández y Anna Sanmartín. 2007. «Redes sociales transnacionales como espacios generadores de desarrollo». Revista Española de Desarrollo y Cooperación (19): 89-105.

Cortés, Almudena y Laura Oso. 2017. «Birds of a Feather in Transnational Flight: Return, Gender and Mobility-Immobility Strategies Between Ecuador and Spain». Revista Española de Sociología (RES) 26(3): 359-372. doi: <https://doi. org/10.22325/fes/res.2017.31>.

Davis, Coralynn. 2015. "Strategic Deployments of «Sisterhood» and Questions of Solidarity at Women's Development Project in Janakpur, Nepal», en Shubhra Gururani y Kim Berry (eds.), Gender in the Himalaya. Feminist Explorations of Identity, Place, and Positionality: 47-73. Katmandú: Association for Nepal and Himalayan Studies.

Díaz de Rada, Ángel. 2010. Cultura, Antropología y otras tonterías. Madrid: Editorial Trotta.

Escobar, Arturo. 1998. La invención del Tercer Mundo: Construcción y deconstrucción del desarrollo. 2007. Caracas: 
Fundación Editorial El perro y la Rana. doi: <https://doi. org/10.1017/СBO9781107415324.004>.

Faist, Thomas y Margit Fauser. 2011. «The Migration-Development Nexus: Toward a Transnational Perspective», en Thomas Faist, Margit Fauser y Peter Kivisto (eds.), The Migration-Development Nexus. A Transnational Perspective: 1-28. Hampshire: Palgrave Macmillan.

Fujikura, Tatsuro. 2001. "Discourses of Awareness». Studies in Nepali History and Society 6(2): 271-313.

Gellner, David N. 2015. "Rituals of Democracy and Development in Nepal», en Siri Hettige y Eva Gerharz (eds.), Governance, Conflict and Development in South Asia: Perspectives from India, Nepal and Sri Lanka, vol. 6: 99-127. Delhi: Sage.

Gil Araujo, Sandra, Laia Costa, Sònia Parella, Leonardo Cavalcanti y Claudia Pedone. 2013. Políticas públicas, migración familiar y retorno de la población migrante latinoamericana en Cataluña: una perspectiva transnacional. Barcelona: Consorci Institut d'Infància i Món Urbà.

Glick Schiller, Nina y Noel B. Salazar. 2013. «Regimes of Mobility Across the Globe». Journal of Ethnic and Migration Studies 39(2): 183-200. doi: <http:doi.org/10.1080/136918 $3 X .2013 .723253>$.

Hamal Gurung, Shobha. 2015. «Coming to America. Gendered Labor, Women's Agency, and Transnationalism», en Nepali Migrant Women: Resistance and Survival in America: 1-83. Nueva York: Syracuse University Press.

Harber, Clive. 2014. Education and International Development: Theory, Practice and Issues. Oxford: Symposium Books.

Headland, Thomas N., Kenneth L. Pike y Marvin Harris. 1990. Emics and Etics. The Insider/Outsider Debate. Londres: Sage.

Kabeer, Naila. 2015. «Gender, Poverty, and Inequality: a Brief History of Feminist Contributions in the Field of International Development». Gender and Development 23(2): 189-205. doi: <https://doi.org/10.1080/13552074.2015.1062300>.

Kern, Alice y Ulrike Müller-Böker. 2015. «The Middle Space of Migration: A Case Study on Brokerage and Recruitment Agencies in Nepal». Geoforum 65: 158-169. doi: <https:// doi.org/10.1016/j.geoforum.2015.07.024>.

King, Russell. 2000. «Generalisations from the History of Return Migration», en Return Migration: Journey of Hope or Despair?: 7-56. Ginebra: United Nations, IOM.

King, Russell y Anastasia Christou. 2011. «Of Counter-diaspora and Reverse Transnationalism: Return Mobilities to and from the Ancestral Homeland». Mobilities 6(4): 451-466. doi: <https://doi.org/10.1080/17450101.2011.603941>.

Korzenevica, Marina. 2016. «Being Included and Excluded: An Exploration of the Impact of Education and Mobility on the Political Engagement of Young Married Nepali Women». Gender, Technology and Development 20(1): 25-48. doi: <https://doi.org/10.1177/0971852415618749>.

Langellier, Kristin M. 1989. «Personal Narratives : Perspectives on Theory and Practice». Text and Performance Quarterly 9(4): 243-276.
Levine, Sarah. 2006. "Getting in, Dropping out, and Staying on: Determinants of Girls' School Attendance in the Kathmandu Valley of Nepal». Anthropology and Education Quaterly 37(1): 21-41. doi: <https://doi.org/10.1525/aeq.2006. 37.1.21>.

Levinson, Bradley y Dorothy Holland. 1996. «The Cultural Production of the Educated Person: An Introduction», en Bradley Levinson, Douglas Foley y Dorothy Holland (eds.), The Cultural Production of the Educated Person: Critical Ethnographies of Schooling and Local Practice: 1-25. Nueva York: State University of New York Press.

Levitt, Peggy. 1998. "Social Remittances: Migration Driven Local-Level Forms of Cultural Diffusion». International Migration Review 32(4): 926-948.

Levitt, Peggy, Josh DeWind y Steven Vertovec. 2006. «International Perspectives on Transnational Migration: An Introduction». International Migration Review 37(3): 565-575. doi: <https://doi.org/10.1111/j.1747-7379.2003.tb00150.x>.

Lind, Birgitte y Jytte Agergaard. 2010. «How Students Fare: Everyday Mobility and Schooling in Nepal's Hill Region». International Development Planning Review 32(3): 311-331. doi: <https://doi.org/10.3828/idpr.2010.11>.

MacDonald, Katie. 2016. "Calls for Educating Girls in the Third World: Futurity, Girls and the 'Third World Woman'». Gender, Place and Culture 23(1): 1-17. doi: <https://doi.org/10 $.1080 / 0966369 \times .2014 .991699>$.

Madawi, Al-Rasheed. 1994. "The Myth of Return: Iraqi Arab and Assyrian Refugees in London». Journal of Refugee Studies 7(2/3): 199-219.

Maharjan, Amina, Siegfried Bauer y Beatrice Knerr. 2012. «Do Rural Women Who Stay behind Benefit from Male Outmigration? A Case Study in the Hills of Nepal». Gender, Technology and Development 16(1): 95-123. doi: <https:// doi.org/10.1177/097185241101600105>

Marcus, George E. 1995. «Ethnography in/of the World System: The Emergence of Multi-Sited Ethnography». Annual Review of Anthropology 24(1): 95-117. doi: <https://doi. org/10.1146/annurev.anthro.24.1.95>.

Maycock, Matthew William. 2017. «Hegemonic at Home and Subaltern Abroad: Kamaiya Masculinities and Changing Mobility in Nepal». Gender, Place and Culture 24(6): 812-822. doi: <https://doi.org/10.1080/0966369X.2015.1058761>.

Ministry of Education, Unicef y Unesco. 2016. All Children in School: Global Initiative on Out-of-School Children. Nepal Country Study. Katmandú: Government of Nepal, Ministry of Education.

Mohanty, Chandra Talpade. 1984. "Under Western Eyes: Feminist Scholarship and Colonial Discourses». Boundary 2 12(3): 333-358. doi: <10.1017/CBO9781107415324.004>.

MOPE. 2016. Nepal Population Report 2016. Katmandú: Government of Nepal, Ministry of Population and Environment.

Mora, Claudia. 2008. «Globalización, género y migraciones». Polis: Revista Latinoamericana 20: 1-11. 
Nightingale, Andrea J. 2011. «Bounding Difference: Intersectionality and the Material Production of Gender, Caste, Class and Environment in Nepal». Geoforum 42(2): 153-162. doi: <https://doi.org/10.1016/j.geoforum.2010.03.004>.

Oso, Laura y Natalia Ribas-Mateos. 2012. «De la sorpresa a la incertidumbre: abriendo etapas en el estudio de la temática sobre género y migración en el contexto español». Papers 97(3): 511-520.

Oso, Laura, Amelia Sáiz-López y Almudena Cortés. 2017. « «Movilidades Cruzadas» en un Contexto de Crisis: Una Propuesta Teórica para el Estudio de la Movilidad Geográfica y Social, con un Enfoque de Género, Transnacional e Intergeneracional». Revista Española de Sociología (RES) 26(3): 293-306

Oxfeld, Ellen y Lynellyn D. Long. 2004. «Introduction: An Ethnography of Return", en Ellen Oxfeld y Lynellyn D. Long (eds.), Coming Home? Refugees, Migrants, and Those Who Stayed Behind: 1-15. Filadelfia: University of Pennsylvania Press.

Parker, Sara. 2012. "Aging in Nepal», en Jason L. Poweell y Sheying Chen (eds.), Aging in Comparative Perspective. Processes and Policies: 59-66. Londres: Springer.

Pascual de Sans, Àngels. 1983. «Connotaciones Ideológicas en el Concepto de Retorno de Migrantes». Papers: Revista de Sociologia 20: 61-71.

Pascual de Sans, Àngels. 1993. "La migración de Retorno en Europa: la Construcción Social de un Mito». Polígonos. Revista de Geografía (3): 89-104.

Pigg, Stacy Leigh. 1992. «Inventing Social Categories Through Place: Social Representations and Development in Nepal». Comparative Studies in Society and History 34(3): 491-513. doi: <https://doi.org/10.1017/S0010417500017928>.

Pigg, Stacy Leigh. 1993. «Unintended Consequences: The Ideological Impact of Development in Nepal». Comparative Studies of South Asia, Africa and the Middle East 13(1-2): 45-58.

Piper, Nicola. 2008. «Feminisation of Migration and the Social Dimensions of Development: The Asian Case». Third World Quarterly 29(7): 1287-1303. doi: <https://doi. org/10.1080/01436590802386427>.

Poertner, Ephraim, Mathias Junginger y Ulrike Müller-Böker. 2011. «Migration in Far West Nepal. Intergenerational Linkages between Internal and International Migration of Ruralto-Urban Migrants». Critical Asian Studies 43(1): 23-47. doi: <https://doi.org/10.1080/14672715.2011.537850>.

Rao, Nitya. 2010. «Migration, Education and Socio-Economic Mobility". Compare: A Journal of Comparative and International Education 40(2): 137-145. doi: <https://doi. org/10.1080/03057920903545973>.

Regmi, G. y C. Tisdell. 2002. «Remitting Behaviour of Nepalese Rural-to-Urban Migrants: Implications for Theory and Policy». The Journal of Development Studies 38(3): 76-94. doi: <https://doi.org/10.1080/00220380412331322351>.

Robinson-Pant, Anna. 2000. Why Eat Green Cucumbers at the Time of Dying. Hamburgo: Unesco Institute for Education.
Robinson-Pant, Anna. 2001. "Development as Discourse: What Relevance to Education?» Compare: A Journal of Comparative and International Education 31(3): 311-328. doi: <https://doi.org/10.1080/03057920120098464>.

Sharma, Jeevan Raj. 2009. «Practices of Male Labor Migration from the Hills of Nepal to India in Development Discourses: Which pathology?» Gender, Technology and Development 12(3): 303-323. doi: <https://doi.org/10.1177/0971852409 01200302>

Sheller, Mimi y John Urry. 2006. "The New Mobilities Paradigm». Environment and Planning A 38(2): 207-226. doi: <https://doi.org/10.1068/a37268>

Shiva, Vandana. 1989. "Development, Ecology and Women», en Vandana Shiva, Stayng Alive, Women, Ecology and Development: 1-13. Trowbridge: Redwood Bks.

Shrestha, Nanda. 1995. "Becoming a Development Agent», en J. Crush (ed.), Power of Development: 266-277. Londres: Psychology Press.

Sibbons, Mo. 1999. «From WID to GAD: Experiences of Education in Nepal», en Christine Hewrad and Sheila Bunwaree (eds.), Gender, Education \& Development. Beyond Access to Empowerment: 189-202. Nueva York: Zed Books Ltd.

Sijapati, Bandita, Ang Sanu Lama, Jeevan Baniya, Jacob Rinck, Kalpana Jha y Amrita Gurung. 2017. Labour Migration and the Remittance Economy. The Socio-Political Impact. Katmandú: Lalitpur.

Sinatti, Giulia. 2011. ««Mobile Transmigrants» or «Unsettled Returnees»? Myth of Return and Permanent Resettlement among Senegalese Migrants». Population, Space and Place 17(2): 153-166. doi: <https://doi.org/10.1002/psp.608>.

Sinatti, Giulia. 2015. «Return Migration as a Win-Win-Win Scenario? Visions of Return among Senegalese Migrants, the State of Origin and Receiving Countries». Ethnic and Racial Studies 38(2): 275-291. doi: <https://doi.org/10.1080/0141 9870.2013.868016>

Skinner, Debra y Dorothy Holland. 1996. «Schools and the Cultural Production of the Educated Person in a Nepalese Hill Community", en Bradley A. Levinson, Douglas E. Foley, Dorothy C. Holland, (eds.), The Cultural Production of the Educated Person: Critical Ethnographies of Schooling and Local Practice: 273300. Nueva York: State University of New York Press.

Tamang, Seira. 2000. «Legalizing State Patriarchy in Nepal». Studies in Nepali History and Society 5(1): 127-156.

Thieme, Susan y Simone Wyss. 2005. «Migration Patterns and Remittance Transfer in Nepal: A Case Study of Sainik Basti in Western Nepal». International Migration 43(5): 60-98.

Unesco. 2000. The Dakar Framework for Action. Dakar: Unesco.

Valentin, Karen. 2012. "The Role of Education in Mobile Livelihoods: Social and Geographical Routes of Young Nepalese Migrants in India». Anthropology \& Education Quarterly 43(4): 429-442. doi: <https://doi.org/10.1111/j.15481492.2012.01195.x.429>.

Valentin, Karen. 2015. «Transnational Education and the Remaking of Social Identity: Nepalese Student Migration to 
Denmark». Identities: Global Studies in Culture and Power 22(3): 318-332. doi: <https://doi.org/10.1080/107028 9X.2014.939186>.

Veale, Angela y Giorgia Dona. 2014. «Complex Migrations, Migrant Child and Family Life Trajectories and Globalisation», en Child and Youth Migration: Mobility-in-Migration in an Era of Globalization: 1-20. Basingstoke: Palgrave Macmillan.

Vega, Cristina. 2016. «El Retorno Más Allá del Mito del Emprendedor. Estrategias Económicas, Familiares y Afectivas de Mujeres y Hombres a su Regreso a Ecuador desde España». Papers 101(4): 415-449. doi: <https://doi. org/10.5565/rev/papers.2182>.
World Economic Forum. 2017. The Global Gender Gap Report 2017. Ginebra.

Yuval-Davis, Nira. 2006. "Intersectionality and Feminist Politics». European Journal of Women's Studies 13(3): 193-209. doi: <https://doi.org/10.1177/1350506806065752>.

Yuval-Davis, Nira. 2016. «Power, Intersectionality and the Politics of Belonging», en Wendy Harcourt (ed.), The Palgrave Handbook of Gender and Development. Critical Engagements in Feminist Theory and Practice: 367-381. Londres: Palgrave Macmillan. 